\title{
Dos nuevas pinturas de Pieter Crijnse Volmarijn en el coleccionismo español
}

\author{
Two new paintings by Pieter Crijnse Volmarijn in the spanish art collecting
}

\author{
Matías Díaz Padrón ${ }^{1}$ \\ Académie Royale d'Archeologie de Belgique \\ Instituto Moll. Centro de investigación en pintura flamenca
}

Resumen: Este artículo se centra en la autoría de Pieter Crijnse Volmarijn en relación a las pinturas Sansón y Dalila y El encuentro final de Helena y Menelao, tradicionalmente vinculadas a Rubens y a su escuela. Respecto a la primera, se expone la influencia de Van Dyck, de Rubens y de la escultura clásica. En cuanto a la segunda, se rechazan las atribuciones a Rubens, sugeridas por Van Puyvelde y Bodart, a la vez que se identifica el tema representado como el reencuentro de Helena con Menelao después de la toma de Troya.

Palabras clave: Volmarijn; Rubens; Van Dyck; Sansón y Dalila; Helena y Menelao

Abstract: This paper focuses on the authorship of Pieter Crijnse Volmarijn related to the paintings Samson and Delilah and The final meeting between Helen and Menelaus, both of them tradicionally linked to Rubens and his school. As far as the first painting is concerned, its influence from Van Dyck and Rubens and also from the classical sculpture is expounded. And as for the second one, its attribution to Rubens, suggested by Van Puyvelde and Bodart, is rejected at the same time that the scene is identified as the reunion between Helen and Menelaus after taking Troy

Key words: Volmarijn; Rubens; Van Dyck; Samson and Delilah; Helen and Menelaus

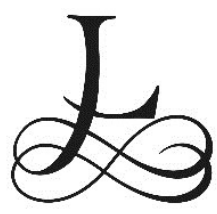

La pintura de Sansón y Dalila (T. 32,6 x 42,3 cm) (fig. 1), aquí tratada, estuvo en The Corcoran Gallery of Art de Washington hasta 2011, momento en que la pinacoteca se

${ }^{1}$ https://orcid.org/0000-0002-5137-7583

(C) 2017 Philostrato. Revista de Historia y Arte 
desprendió de ella para solventar parte de sus problemas financieros ${ }^{2}$. La obra salió a subasta catalogada como círculo de Anton van Dyck ${ }^{3} \mathrm{y}$, en este sentido, no es difícil ver en ella la influencia de la tabla de Rubens de la National Gallery de Londres (inv. NG6461) (fig. 2), divulgada por el grabado de Jacob Matham (fig. 3). Nicolaas Rockox, burgomaestre de Amberes, encargó esta última al maestro poco después de su regreso de Italia en 1608. Su historia externa es sobradamente conocida para entrar en más detalles ${ }^{4}$. La espalda de Sansón se impone en el plano, al igual que la dramática tensión que se presagia. Es evidente la relación con el Torso del Belvedere de los Museos Vaticanos (inv. 1192), que Rubens había dibujado y que tuvo gran repercusión entre discípulos tales como Anton van Dyck, Pieter Soutman y Christiaen van Couwenbergh, por citar tres de los ejemplos más destacados 5 .

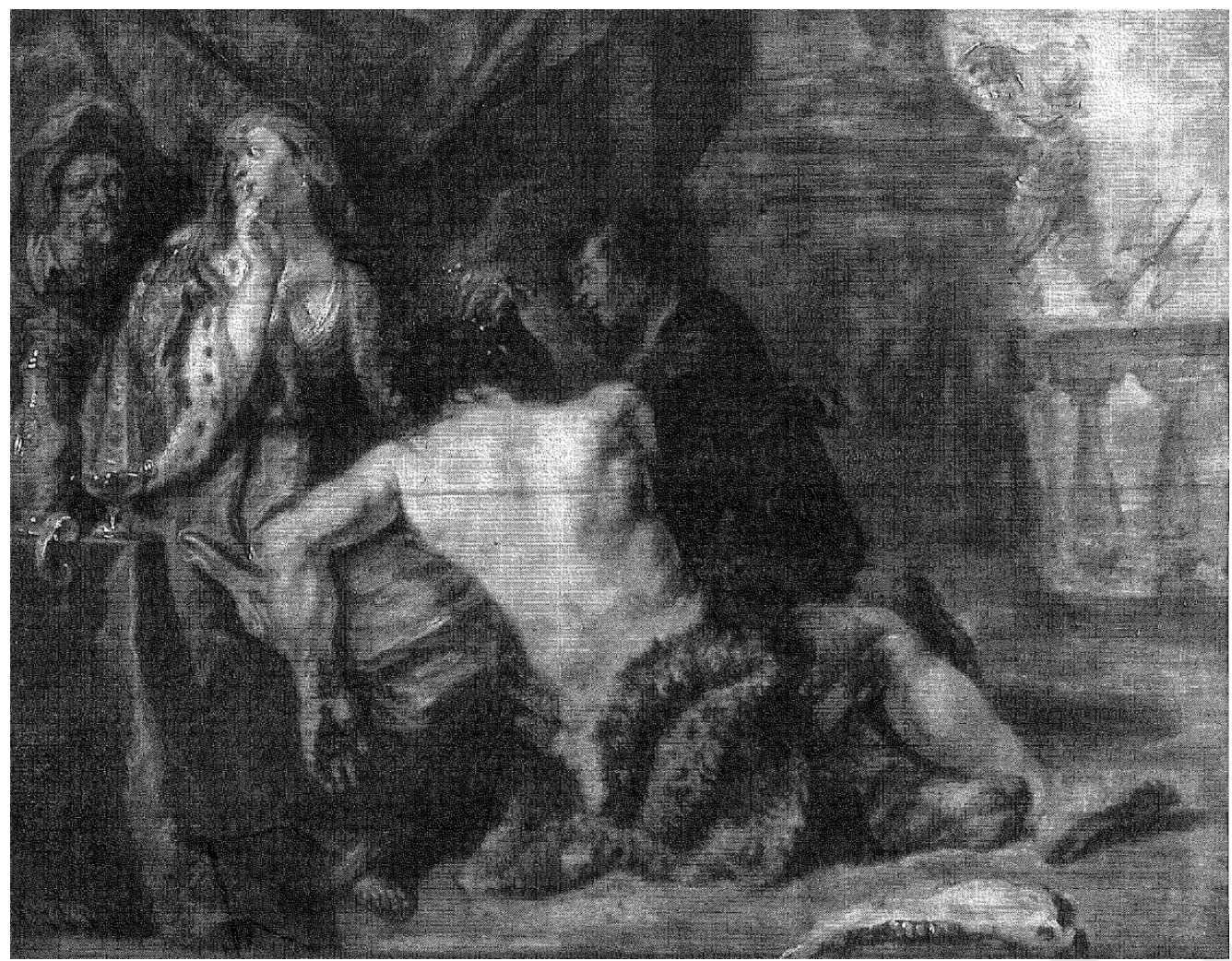

Fig. 1. Pieter Crijnse Volmarijn, Sansón y Dalila, procedente de The Corcoran Gallery of Art, Washington

Ese modelo también influirá en Pieter Crijnse Volmarijn, a quien atribuyó esta obra a la que el pintor incorpora su personal factura y su exagerado gusto por los ornatos. Representa la espalda de Sansón de frente y deja laxo el brazo izquierdo, rozando el pavimento de la estancia. Muestra la parte trasera de la cabeza, cuyos cabellos corta un sirviente y cuyo rostro queda oculto, lo que distancia a Volmarijn de Rubens, no así la disposición de las piernas ni la distribución de los distintos personajes de la escena. En el lado derecho de la obra de Volmarijn, un soldado se aproxima a la balaustrada,

\footnotetext{
2 Vid. "Cierra la histórica galería de arte 'Corcoran Gallery Art"', El Mundo, 16 de mayo de 2014.

3 Chistie's Nueva York, 26-I-2011, no 138.

${ }^{4}$ Roger-Adolf d'Hulst y Marc Vandenven, Corpus Rubenianum Ludwig Burchard. The Old Testament, III, (London: Harvey Miller Publishers, 1989), no 31, pp. 107-113.

${ }^{5}$ Ibidem, p. 112.
} 
bajo un cielo abierto a la luz del atardecer. En cambio, el maestro opta por una puerta en la oscuridad de la noche. A los pies de Sansón, está la quijada de burro causante del suplicio que está por venir, un detalle ausente en el original de Rubens, quien cubrió ese espacio con una rica alfombra plegada. Renunciando a esta lujosa decoración, Volmarijn simplifica el fondo con un muro liso y el plinto de una robusta columna. Así, este pintor rompe con la distribución lineal y en perfil de la fuente que le sirve de inspiración, para girar el cuerpo de Sansón y exponer su espalda al espectador. Estas diferencias continúan con Dalila, vista aquí de frente y con el dedo índice en sus labios, imponiendo silencio a la vieja sirvienta en la penumbra. Igual que en Rubens, la luz baña a Dalila y la espalda de Sansón, desde un punto alto situado a la izquierda, lo que no impide otro foco de luz del exterior, en el la-

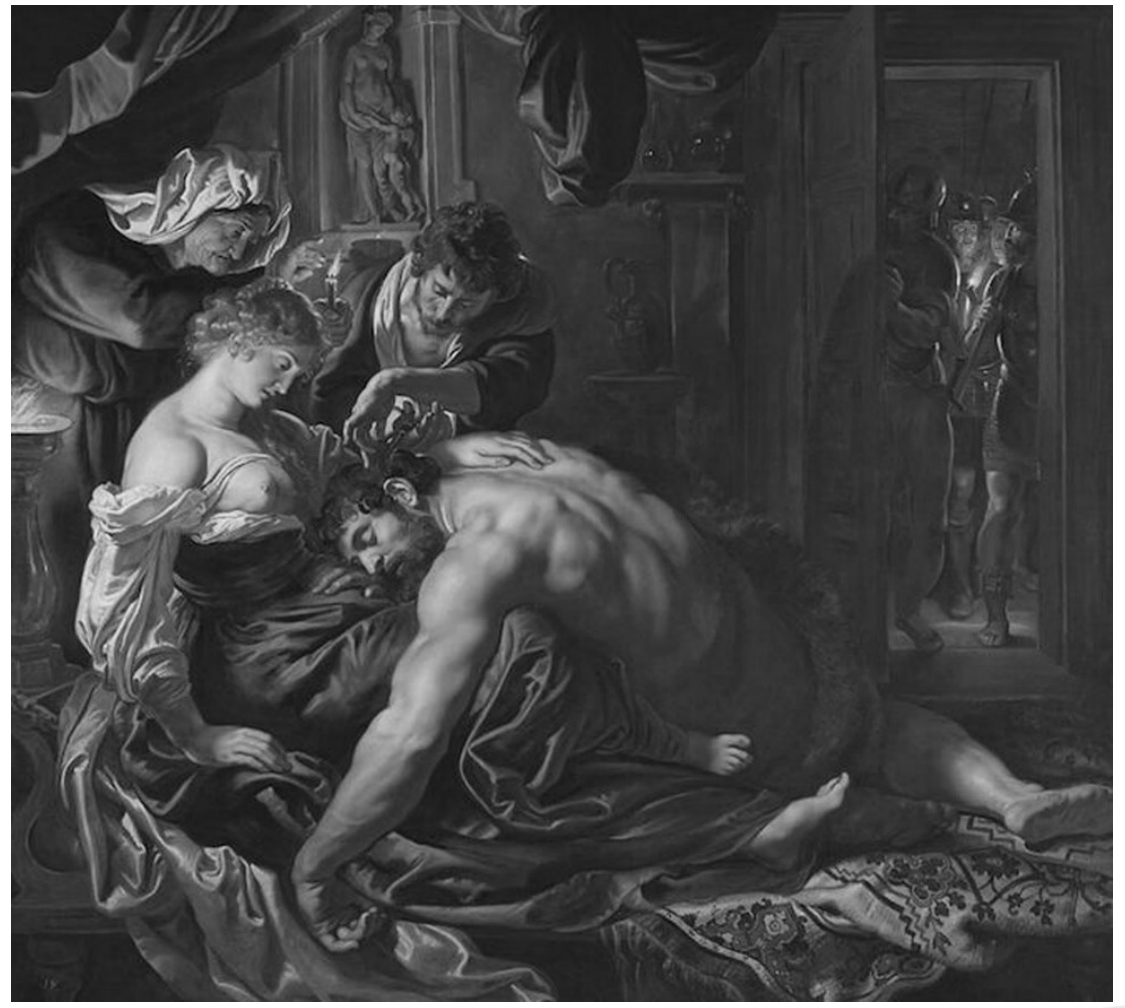

Fig. 2. Peter Paul Rubens, Sansón y Dalila, National Gallery of Art, Londres

do derecho, por donde asoma el ya citado soldado, en espera del corte de las trenzas del héroe.

El cuerpo de Sansón se inspira en el Galo moribundo de los Museos Capitolinos (inv. MC0747) (fig. 4), escultura que gozó de gran prestigio entre los artistas y que también sirvió de prototipo a Van Dyck y a Soutman ${ }^{6}$. Como ya se adelantó, Volmarijn tuvo la habilidad de ocultar el rostro de Sansón, dejando a la imaginación el dramático momento, a semejanza de lo que hiciera Van Dyck en su versión de la Dulwich Picture Gallery de Londres (inv. DPG127) (fig. 5) y Velázquez en Mercurio y Argos del Museo Nacional del Prado (inv. P01175). La monumentalidad y la amplitud espacial son rasgos

${ }^{6}$ Francis Haskell y Nicholas Penny, Taste and the Antique: The Lure of Classical Sculpture 1500-1900, (New Haven and London: Yale University Press, 1981), 224-227. 
tomados de la obra de Rubens. Sin embargo, el giro del torso y otros detalles expresivos los debe Volmarijn a Van Dyck en la citada versión de la Dulwich Picture Gallery de Londres, invirtiendo a los protagonistas en la escena y, con más proximidad, al dibujo preparatorio fechado en 1618-1619, que se conserva en el Kupferstichkabinett de Berlin (KdZ 5396)7 (fig. 6).

Sansón está cubierto con una espesa piel mientras Dalila impone silencio con el dedo, un detalle aparentemente accidental que no está en Rubens, como tampoco está la posición frontal de la espalda de Sansón. Esta última fue ensayada por Van Dyck en un dibujo de la Kunsthalle de Brême (fig. 7), fiel a Rubens en el diseño de Sansón de espaldas y con el brazo caído. Este dibujo de la Kunsthalle sirvió de inspiración a Volmarijn que, al mismo tiempo,

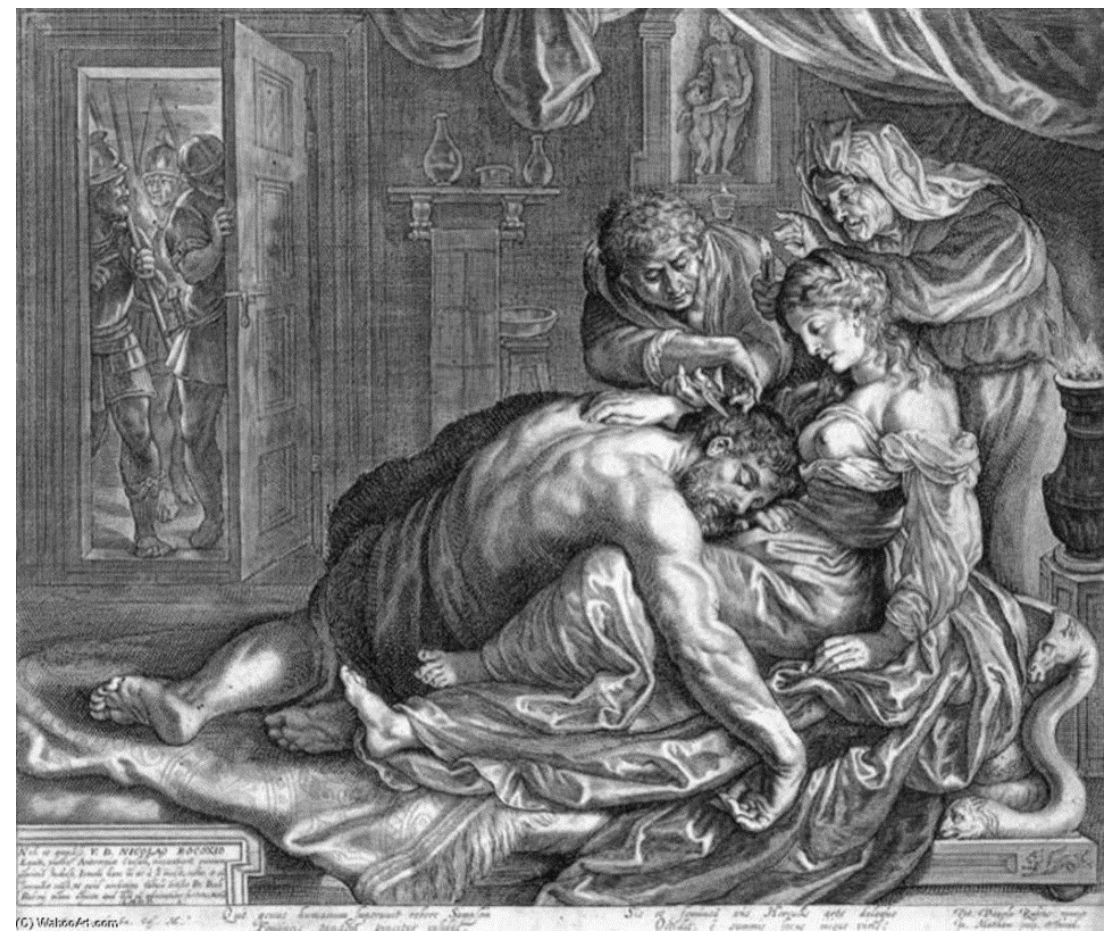

Fig. 3. Jacob Matham siguiendo a Rubens, Sansón y Dalila, grabado

tomó del anteriormente referido dibujo de Berlín el muro frontal con la robusta columna y el pilar. En éste es especialmente interesante la balaustrada, omitida por Van Dyck en el lienzo definitivo de la Dulwich Picture Gallery, pero representada en la pintura aquí tratada, evidenciando que Volmarijn conoció los dibujos de su colega. El personal dibujo de la espalda fue ensayado por Van Dyck en el lienzo de San Martín dando su capa a los pobres, actualmente localizado en Windsor Castle como parte de The Royal Collection of Her Majesty Queen Elizabeth II (inv. RCIN405878) ${ }^{8}$, en el que está siguiendo un boceto poco conocido de Rubens en el comercio londinense.

La composición de la obra aquí estudiada revela la dramática lucha de los israelitas con los filisteos, en la que la fuerza se opuso a la inteligencia. La

\footnotetext{
${ }^{7}$ Horst Vey, Die Zeichnungen Anton van Dycks, I, (Bruxelles: Arcade, 1962), n03; Christopher Brown, Van Dyck Dessins, (New York: The Pierpont Morgan Library, 1991), no 9, pp. 71-72.

${ }^{8}$ Sotheby's, 2-VII-1986, no 139.
} 
quijada de asno, que no aparece ni en Rubens ni en Van Dyck, rememora tanto los hechos que desencadenaron el instante representado como la fuerza de Sansón ${ }^{9}$. Éste, atado y entregado a los filisteos, había roto las ligaduras y había matado a mil hombres con la quijada (Jue 15, 1-17). Los filisteos confiaron entonces en Dalila para conocer la debilidad de Sansón, quien desveló el secreto de su imponente fuerza tras mentir previamente en tres ocasiones: "... Y le abrió de par en par su corazón, diciendo: «Nunca ha tocado la navaja mi cabeza, pues soy nazareo de dios desde el vientre de mi madre. Si me rapasen, perdería mi fuerza, quedaría débil y sería como todos los hombres». Dalila vio que en verdad le había abierto de par en par su corazón; y mandó llamar a los príncipes de los filisteos..." (Jue 16, 4-20) ${ }^{10}$.

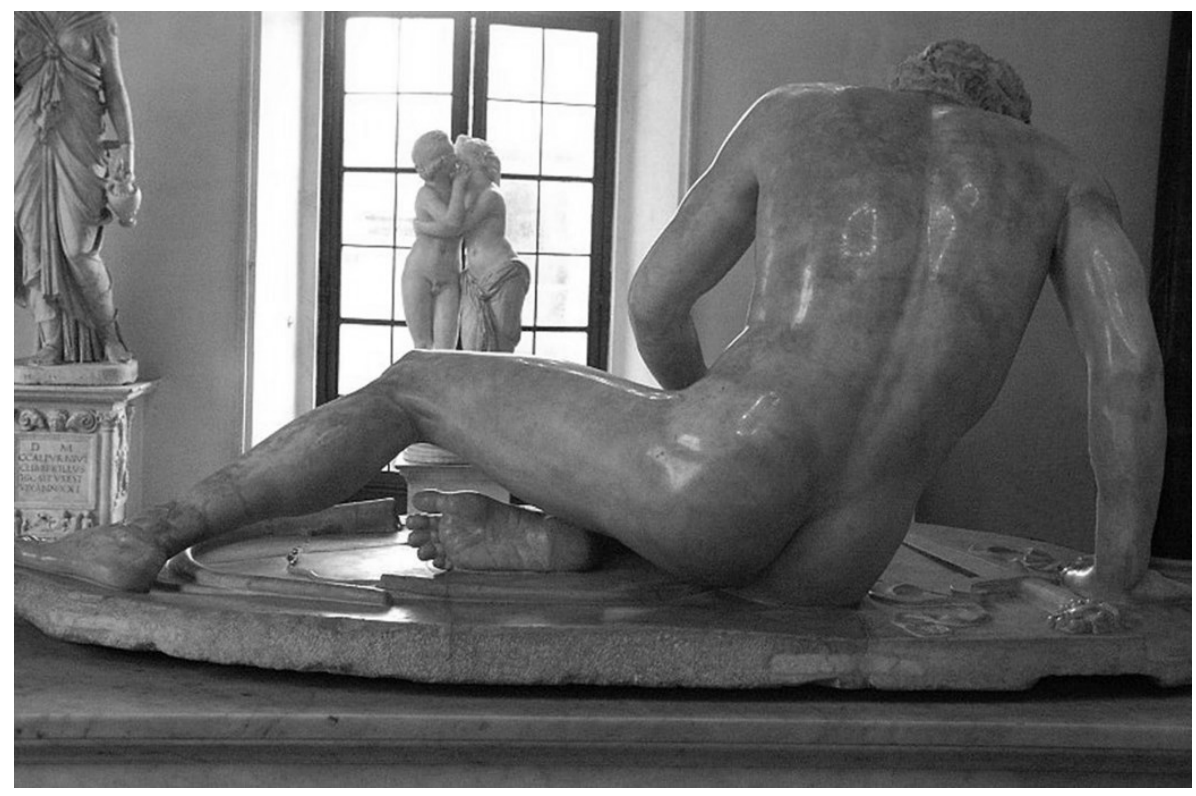

Fig. 4. Escultura de Galo moribundo o Galata Capitolino, Museos Capitolinos, Roma.

La influencia de Rubens y de Van Dyck es habitual en la escasa producción conocida de Volmarijn, si bien su estudio se reduce a unas pocas publicaciones de las últimas décadas ${ }^{11}$. Sigue modelos de Rubens en el

\footnotetext{
${ }_{9}^{9}$ Pieter Crijnse Volmarijn fue fiel a las consignas de la Contrarreforma en el norte, donde la victoria de Sansón sobre los filisteos se comparaba con David venciendo a Goliat y se consideraba prefiguración de la resurrección de Cristo. Por ello, este episodio de la historia de Sansón es interpretado como el triunfo de Jesús sobre Satán. Vid. Louis Reau, Iconographie de L'Art Chrétien. Iconographie de la Bible: Ancien Testament, II, 1, (París: Presses Universitaires de France, 1957), pp. 243; John B. Knipping, Iconography of the Counter Reformation in the Netherlands. Heaven on earth, I, (De Graff, 1974), p. 196; Ibidem, II, pp. 22, 26 y 47.

10 Vid. L. Reau, Iconographie de L'Art Chrétien, Op. Cit., pp. 245-246.

${ }^{11}$ Pieter Haverkorn Van Rijsewijk, "Rotterdamsche Schilders: De schilders Volmarijn", Oud Holland, nº12, (1894), pp. 136-159; Sturla J. Gudlaugsson, "Crijn Hendricksz Volmarijn, een Rotterdamse Caravaggist", Oud Holland, no 4, vol 67, (1952), pp. 241-247; Roger-Adolf d'Hulst, "Pieter Crijnse Volmarijn, een Rotterdamse navolger van de Antwerpse schilderschool uit de 17de eeuw", Mededelingen van de Koninklijke Vlaamse Academie voor Wetenschappen, Letteren en Schone Kunsten van België, 32, Brüssel, (1970), pp. 1-22; Julius S. Held, "Further Data on Pieter Crijnse Volmarijn", en Anne-Marie Logan, Essays in Northern European Art presented to Egbert Haverkamp-Begemann on his $60^{\text {th }}$ birthday, (Doornspijk: Davaco, 1983), pp. 102-105; Roger-Adolf d'Hulst, "Een paar toevoegingen aan het oeuvre van Pieter Crijnse Volmarijn", en Logan, Essays in Northern European Art, Op. Cit., pp. 113-116; Nora Schadee et al., Rotterdamse meesters uit de Gouden Eeuw, (Rotterdam: Historisch Museum, 1994); Matías Díaz Padrón, "Un boceto de Pieter Crijnse Volmarijn recuperado del anonimato en España", Tendencias del Mercado del Arte, n²7, (2009), pp. 40-41; Matías Díaz Padrón, "Dos lienzos identificados de Pieter Crijnse Volmarijn en el Ermitage de San Petersburgo y Galeria de los Uffizi de Florencia", Archivo Español de Arte, no 335, vol. 84, (2011), pp. 276-282.
} 
Encuentro de José con sus hermanos de la colección de Paul Cassirer en Berlín, pero fiel a su singular personalidad, desgarrada e indiferente al rico cromatismo rubeniano y a la refinada estética de Van Dyck. Algún modelo de este último utiliza para El Jardín del Amor de la colección John Bass de Estados Unidos y el rostro de Dalila lo repite en Meleagro y Atalanta de la colección Held ${ }^{12}$ (fig. 8). Es habitual en su producción el fondo sombrío y oscuro, con sugestiones de Jacob Jordaens, con quien a veces se confunde. No faltan lujosos ornamentos "orientales" ni orfebrería, tales como el manto de armiño que viste Dalila y el rico alfombrado, frecuentes en su obra. La visión del espacio está en la frontera de la estética holandesa, una interesante categoría que se comprende por el nacimiento de Volmarijn en Rotterdam ${ }^{13}$.

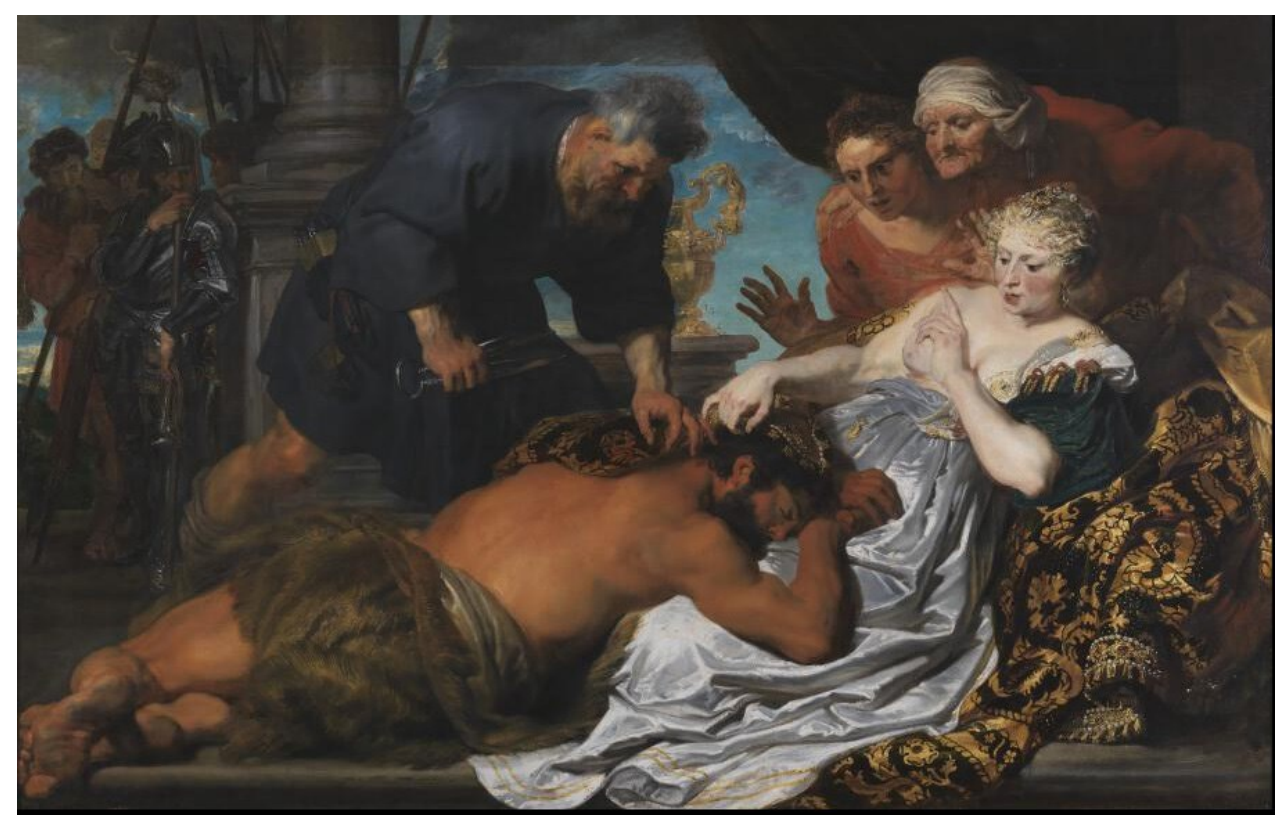

Fig. 5. Anton Van Dyck, Sansón y Dalila, Dulwich Picture Gallery, Londres

En fin, como acierta a decir Held, "como luces fantasmales, las pinturas y dibujos de este artista aparecen para burlar y guiar por mal camino a los estudiosos de la pintura flamenca y, como esas escurridizas luces, suelen desaparecer de la vista después de haber hecho una breve aparición en catálogos de subastas o en colecciones privadas..."14.

Al mismo maestro se puede adscribir una pintura de notable tamaño ( $L$. $200 \times 180 \mathrm{~cm}$.) del coleccionismo madrileño, pero procedente de fuera de España, a juzgar por la documentación existente, que siempre la atribuye a Pedro Pablo Rubens y la identifica con el tema de Rapto de Helena de Troya por Paris (fig. 9). Es obra de calidad y buen estado de conservación. Cuenta

\footnotetext{
12 Held, "Further Data on Pieter Crijnse Volmarijn", Op. Cit., pp. 103-104. Vendida por Christie's Nueva York, 27-I-2009, no 31.

13 d'Hulst, "Pieter Crijnse Volmarijn, een Rotterdamse Navolger van de Antwerpse Schilderschool uit de 17de Eeuw", Op. Cit., 1970, p. 1; Haverkorn Van Rijsewijk; Gudlaugsson;... Vid. Nota 9.

14 "Like so many will-o'-the-wisps, paintings and drawings by this artist appeared to tease and lead astray the students of Flemish painting, and like those elusive lights, they often disappeard from sight after having made a brief appearance in auction catalogues or private collections..." Vid. Held, "Further Data on Pieter Crijnse Volmarijn", Op. Cit., p. 102.
} 
con referencias en exposiciones y en estudios aislados, tanto en España como en el extranjero. Especialmente importante es el informe de Leo van Puyvel-

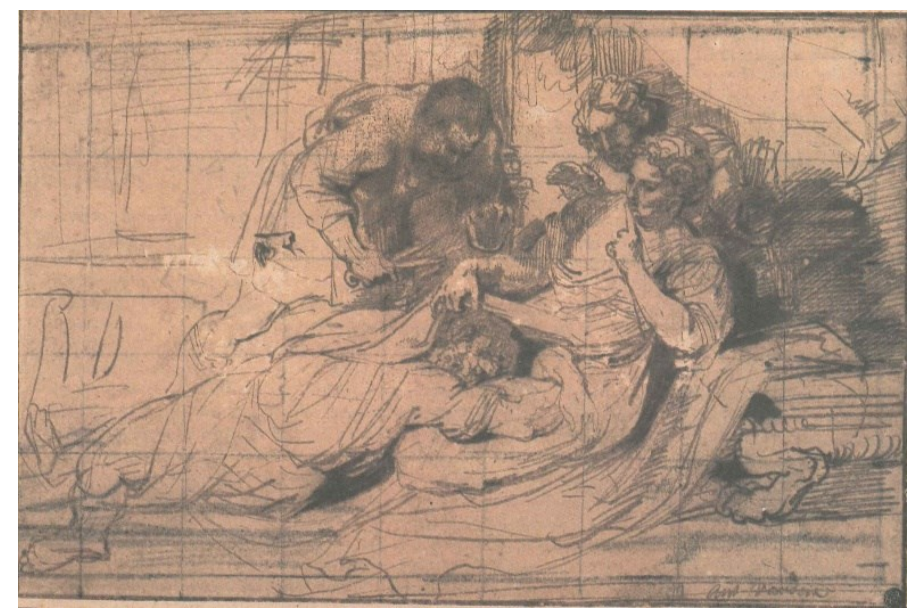

Fig. 6. Anton Van Dyck, Sansón y Dalila, dibujo, Kupferstichkabinett, Berlín

de, atribuyéndola a Rubens el 5 de octubre de 1960. Este estudioso apoya su tesis en un boceto referenciado en el inventario de Jacob Horemans, vecino de Amberes, el 7 y 8 de mayo de $1678^{15}$.

Sin embargo, no se conoce ninguna obra de Rubens con este asunto, salvo el boceto citado en 1678 por Jan Denucé: "Leene Schetse van Rubens wesende d'Ontschakinge van Helena" [un boceto de Rubens referente al rapto de Elena $]^{16}$. La opinión de Van Puyvelde fue seguida por especialistas en pin-

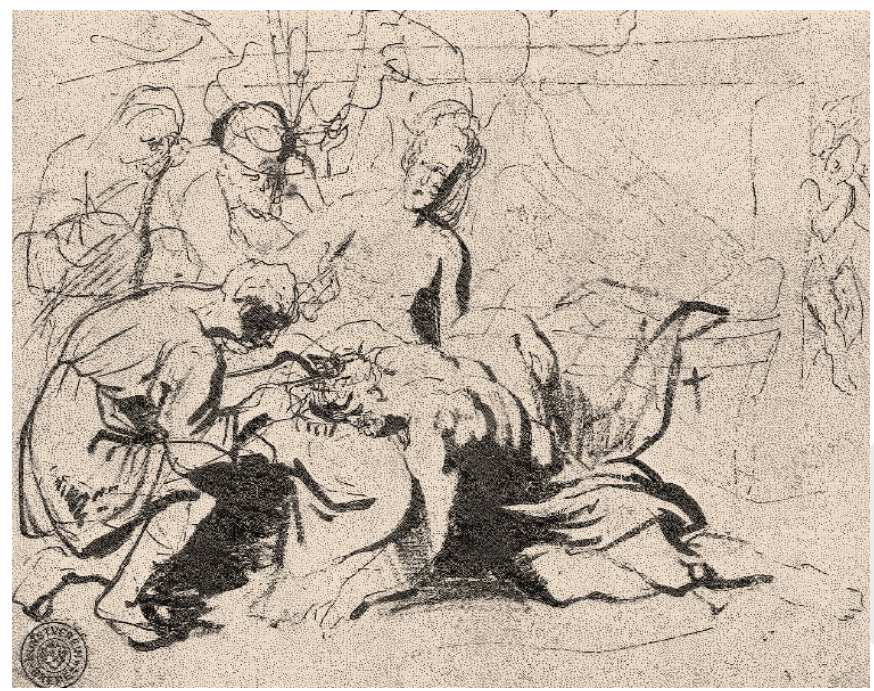

Fig. 7. Anton Van Dyck, y Dalila, dibujo, Kunsthalle, Brême

\footnotetext{
${ }^{15}$ En el informe consta: "The picture (canvas, 76-70 inches) reproduced on the back and representing, The Rape of Helen of Troy, is in my opinion a genuine work by Peter-Paul Rubens, made soon after his settlement in Antwerp. A picture by Rubens with such a subject is mentioned in the inventory of the succession of Jacob Horremans 1678 in Antwerp. (Archives of the city of Antwerp). Brussels, 5 october 1960. Leo van Puyvelde". SAA, Felixarchief.

16 Jan Denucé, De Antwerpsche "Konstkamers". Inventarissen van kunstverzamelingen te Antwerpen in de 16e en 17e eeuwen, (Amsterdam: De Spiegel, 1932), p.231.
} 
tura flamenca, tales como Carlos Catalán ${ }^{17}$ y el profesor Didier Bodart, en más de una monografía de Rubens y en catálogos de exposiciones alusivas al maestro flamenco ${ }^{18}$.

Bodart estima posible la participación de algún destacado discípulo, como Jordaens o Van Dyck: "L'esecuzione veloce lascia pensare che il pittore si sia servito di qualche aiutante del suo atelier, che, a quel tempo, comprendeva, tra gli altri, Anton van Dyck e Jacob Jordaens."19. Hasta aquí el estado de la cuestión. No encuentro razones para mantener esta tesis, pues los certificados hacen referencia a un dibujo y a un grabado de los que no consta testimonio visual alguno $y$, a pesar de lo expuesto hasta ahora, no se conoce pintura con este asunto en la copiosa producción de Rubens.

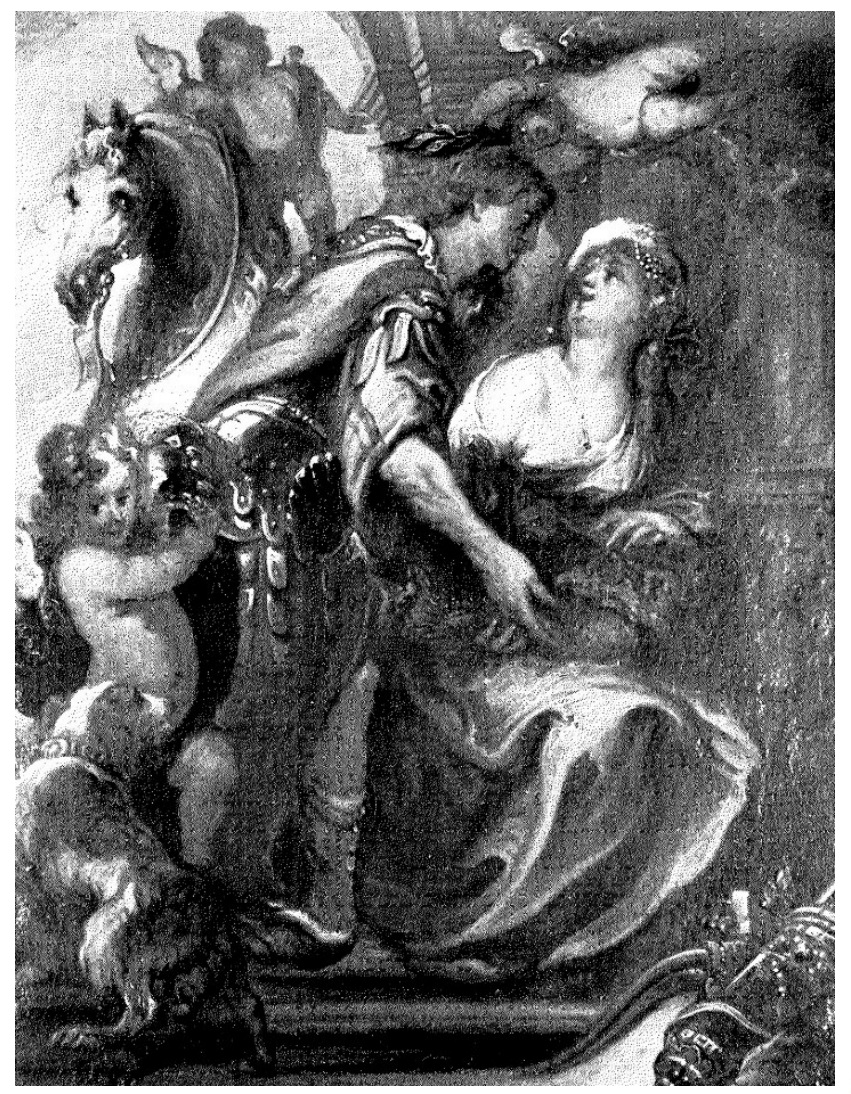

Fig. 8. Pieter Crijnse Volmarijn, Meleagro y Atalanta, antigua colección Held. Paradero desconocido.

Helena, hija de Zeus y Leda, es la protagonista de la guerra de Troya en La Ilíada. El filósofo Plutarco sostuvo que fue raptada por Teseo y Pirítoo cuando danzaba en el templo de Artemisa Ortia. En la huída, Teseo decidió tomarla por esposa, pero fue rescatada por sus hermanos, Cástor y Pólux, antes de que aquél lograra ver cumplido su deseo. Su belleza atrajo a los nobles de la Hélade. Ella eligió a Menelao y se casaron, pero Paris se opuso, la raptó y convivieron durante veinte años en Troya. El rapto de Helena desencadenó el

17 Certificado firmado y fechado en Etxauri, el 5 de junio de 1998

18 Didier Bodart, Rubens, (Milán: Carroggio, 1981),no 419, p. 173; Didier Bodart, Rubens, (BarcelonaVerona: Arnoldo Mondadori-Carroggio Ediciones, 1983); Rubens e la pittura fiamminga del '600, ed. Didier Bodart (Sardegna: Cagliari Cittadela dei Musei, 1990), no 8., pp. 116.

${ }^{19}$ Rubens e la pittura fiamminga del '600, Cat. Exp., 1990, pp. 26. 
enfrentamiento entre aqueos y troyanos, el cual se prolongó durante diez años ante las murallas de la ciudad. En La Ilíada, Homero ignora el rapto de Helena por Teseo y Pirítoo. Siguiendo a este poeta, los aqueos la reclamaron para devolverla a Menelao, que no la había olvidado y ella se vio en la encrucijada de traicionar a los troyanos, o bien a los aqueos.

Hasta aquí, nos vemos implicados en dos raptos. Un posterior encuentro, más que otro rapto, podría corresponder a lo que narra esta pintura. La clave del contenido está en la espada con la que un guerrero amenaza a Helena mirándola de frente. Éste es Menelao, lo que abre las puertas del contenido real de la pintura, que hasta ahora se ha tenido por el rapto de Helena por Paris.

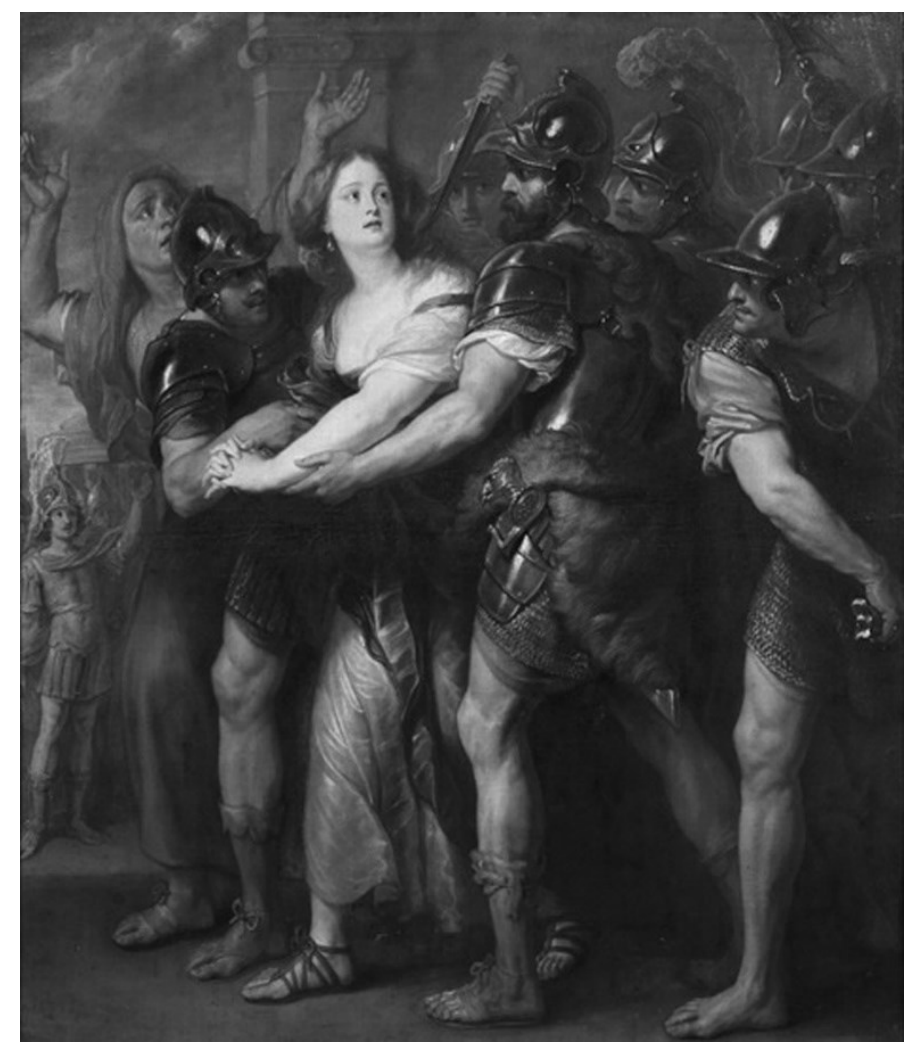

Fig. 9. Pieter Crijnse Volmarijn, El encuentro final de Helena y Menelao, colección privada, Madrid

Helena había pasado por los brazos de Teseo, Menelao y Paris. El caso es que Menelao vuelve a su vida. Desde Troya, Helena había avisado con una antorcha a los aqueos, que acampaban con Menelao a los pies de la muralla. Al irrumpir estos en la ciudad, Helena, confiada, se aproximó y la aprisionaron: "Cuando Menelao llega, levanta la espada para matarla, pero al verla medio desnuda la espada cae de la mano del marido engañado. Los griegos, entonces, quisieron lapidarla, pero de nuevo la belleza de su cuerpo la salvó" ${ }^{20}$. Esto es lo que narra la pintura. Helena es una mujer que, aunque constantemente amenazada, sabe que su belleza la sacará de todos los malos

${ }^{20}$ Agustí Bartra, Diccionario de mitología, (Barcelona:Grijalbo, 1982), pp. 82-83. 
pasos. Termina la guerra de Troya y los dos, Menelao y Helena, regresan a Esparta, donde ella fue un ejemplo de las virtudes domésticas.

El origen de la pintura no va más allá del boceto citado por Leo van Puyvelde en 1678 , un testimonio que tampoco es válido para este lienzo que atribuye a Rubens. La mención más antigua a esta pintura permite situarla en la colección florentina de Enrico Frascione, en 1880. Por herencia, en 1934 pasó a la colección de Vittorio Frascione, también en Florencia. Desde aquí se integró en el coleccionismo madrileño a nombre de Rubens, sin otra opinión que rompiera con esta atribución. En fin, lo razonado en estas líneas desestima la atribución del lienzo a Rubens y precisa el momento crucial de la accidentada existencia de la mujer más bella del mundo homérico. 
Bibliografía:

Bartra 1982: Agustí Bartra, Diccionario de mitología, (Barcelona:Grijalbo, 1982).

Bodart 1981: Didier Bodart, Rubens, (Milán: Carroggio, 1981).

Bodart 1983: Didier Bodart, Rubens, (Barcelona-Verona: Arnoldo MondadoriCarroggio Ediciones, 1983).

Bodart 1990: Rubens e la pittura fiamminga del '600, ed. Didier Bodart (Sardegna: Cagliari Cittadela dei Musei, 1990).

D'Hulst 1970: Roger-Adolf d'Hulst, "Pieter Crijnse Volmarijn, een Rotterdamse navolger van de Antwerpse schilderschool uit de 17de eeuw", Mededelingen van de Koninklijke Vlaamse Academie voor Wetenschappen, Letteren en Schone Kunsten van België, 32, Brüssel, (1970), pp. 1-22.

D'Hulst Roger-Adolf d'Hulst, "Een paar toevoegingen aan het oeuvre van Pieter Crijnse Volmarijn", en Logan, Essays in Northern European Art, Op. Cit., pp. 113-116.

D'Hulst, Vandenven 1989: Roger-Adolf d'Hulst y Marc Vandenven, Corpus Rubenianum Ludwig Burchard. The Old Testament, III, (London: Harvey Miller Publishers, 1989).

Denucé 1932: Jan Denucé, De Antwerpsche "Konstkamers". Inventarissen van kunstverzamelingen te Antwerpen in de $16 \mathrm{e}$ en $17 \mathrm{e}$ eeuwen, (Amsterdam: De Spiegel, 1932).

Díaz Padrón 2009: Matías Díaz Padrón, "Un boceto de Pieter Crijnse Volmarijn recuperado del anonimato en España", Tendencias del Mercado del Arte, n027, (2009), pp. 40-41.

Díaz Padrón 2011: Matías Díaz Padrón, "Dos lienzos identificados de Pieter Crijnse Volmarijn en el Ermitage de San Petersburgo y Galeria de los Uffizi de Florencia", Archivo Español de Arte, no 335, vol. 84, (2011), pp. 276-282.

Gudlaugsson 1952: Sturla J. Gudlaugsson, "Crijn Hendricksz Volmarijn, een Rotterdamse Caravaggist", Oud Holland, no 4, vol 67, (1952), pp. 241-247.

Haskell, Penny 1981: Francis Haskell y Nicholas Penny, Taste and the Antique: The Lure of Classical Sculpture 1500-1900, (New Haven and London: Yale University Press, 1981).

Held 1983: Julius S. Held, "Further Data on Pieter Crijnse Volmarijn", en Anne-Marie Logan, Essays in Northern European Art presented to Egbert Haverkamp-Begemann on his $60^{\text {th }}$ birthday, (Doornspijk: Davaco, 1983), pp. 102-105.

Kinipping 1974: John B. Knipping, Iconography of the Counter Reformation in the Netherlands. Heaven on earth, I, (De Graff, 1974). 
Reau 1957: Louis Reau, Iconographie de L'Art Chrétien. Iconographie de la Bible: Ancien Testament, II, 1, (París: Presses Universitaires de France, 1957).

Schadee 1994: Nora Schadee et al., Rotterdamse meesters uit de Gouden Eeuw, (Rotterdam: Historisch Museum, 1994).

Van Rijsewijk 1894: Pieter Haverkorn Van Rijsewijk, "Rotterdamsche Schilders: De schilders Volmarijn", Oud Holland, n¹2, (1894), pp. 136-159.

Vey 1991: Horst Vey, Die Zeichnungen Anton van Dycks, I, (Bruxelles: Arcade, 1962), n03; Christopher Brown, Van Dyck Dessins, (New York: The Pierpont Morgan Library, 1991). 